\title{
ENGINEERING
}

\section{Convinced Anomaly Vent The Probableof Plant Hereditary Supplies For Food And Agriculture}

\author{
Prof. Giuseppe Santi, Prof. Laura Campania \\ Plant Production And Protection Division, Food And Agriculture Organization \\ Mediterranea University Of Reggio Calabria, Italy
}

\begin{abstract}
The choices for increasing food production by a minimum of seventieth over consecutive four decades thus on keep up with a apace increasing human population area unit bedeviled by erratic weather conditions, depleted tillable lands, dwindling water supplies and by the many environmental and health prices for increasing the employment of agrochemicals. increased productivities through "smart" crop varieties that yield additional with fewer inputs could be a viable possibility. However, the hereditary similarities amongst crop varieties-which render entire cropping systems susceptible to constant stresses-coupled with unvarying parental materials limit the probabilities for uncovering novel alleles of genes and, hence, collecting new cistron mixtures to interrupt yield plateaux and enhance resilience. iatrogenic mutation unmasks novel alleles that area unit controlled to breed superior crop varieties. The historical antecedents, theoretical and sensible issues, and also the successes of iatrogenic anomaly in crop improvement area unit reviewed at the side of however iatrogenic cause underpins plant genomics. The roles of cell and biology techniques in enhancing the efficiencies for the induction, detection and readying of mutation events also are reviewed. Also, the mixing of phenomics into iatrogenic cause and also the use of pre-breeding for facilitating the incorporation of mutants into crop improvement area unit advocated.
\end{abstract}

Keywords: iatrogenic anomaly; mutagens; mutant; crop improvement; genomics; cell biology; TILLING; phenomics; pre-breeding

Addressing the Challenge of Feeding the twenty first Century World 


\section{ENGINEERING}

A most important challenge to crop production is that the attainment of increased productivities in farmer's fields, particularly in developing countries. The Food and Agriculture Organization of the international organization (FAO) calculable that seventieth additional food is required to feed adequately the over 9 billion individuals expected to inhabit planet earth by 2050 [1]. The task of meeting this target, which needs a thirty seventh increase over the historical annual progressive rates [2], is clearly as intimidating because the scope of the envisaged will increase is unexampled. The difficulties area unit combined by the finite nature of tillable lands and agricultural water resources. Indeed, in most elements of the world-including the critically food insecure regions of the developing world [3-6], these supplies area unit either stagnant or area unit dwindling on accounts of extra demands for them by the demographic and economic pressures of urbanization and also the competitive wants for manufacturing farm animal feeds, bioenergy production different|and several other\} other industries [1]. the results of temperature change and variations also are rendering vital areas of tillable lands unsuitable for crop production [7-9]; this is often a significant driver for food insecurity.

FAO [11] counseled that, for realizing the imperative of the low-input agriculture being proffered for the twenty first Century, farmers need a collection of improved crop varieties that area unit hereditary ally various, temperature change resilient, input use-efficient, high yielding, have increased organic process and different quality attributes and are bred for adaptation to a variety of agroecosystems and farming practices. But, the envisaged hereditary ally various portfolio of appropriate crop varieties area unit neither offered to farmers $[2,12,13]$ nor do the present breeding ways hold promise for delivering them [14]. The very slender hereditary base of the offered kinds of crops and also the parental lines for breeding new ones nullify efforts to reinforce productivities in farmers' fields, increase vulnerabilities and thereby exist food security.

\section{Mutation}

Mutation is that the ancestral modification to the hereditary material; people that manifest changed characteristics on account of ancestral changes area unit called mutants. In nature, the mixtures of the errors that occur throughout the replication of polymer (DNA) associate degreed damages to the current hereditary material on account of an individual's exposure to daylight and ultra-violet (UV) radiation and diseases result in ancestral changes to the hereditary blueprint. different forms of anomaly embrace duplications whereby whole elements of the order area unit doubled and translocations whereby a region of a body is transferred to a nonhomologous chromosome; these occur as a results of errors throughout cell division cellular 


\section{THE AMERICAN JOURNAL OF}

\section{ENGINEERING}

division. Their effects rely on whether or not or not, and how, genes area unit affected in such anomaly. The accumulations of such aberrations are the primer for evolution and evolution. Stretches of polymer conjure the essential unit of heredity, the gene. The refined changes to polymer sequences area unit the foremost helpful for crop improvement because the additional gross alterations (at the body and cellular levels) invariably confer some levels of unfitness and even morbidness. a method for classifying anomaly at the polymer sequence level is to categorise the mutation on the premise of however the modification affects the gene's ability to synthesize the macromolecule that it's liable for. In general, the anomaly that area unit necessary in crop improvement typically involve single bases and should or might not have an effect on macromolecule synthesis.

\section{The common forms of anomaly in polymer sequences include:}

Single Base Substitutions or purpose Anomaly

One base is replaced by another may well be either transitions or transversions. in a very transition, one purine ( $\mathrm{A}$ or $\mathrm{G}$ ) or pyrimidine ( $\mathrm{C}$ or $\mathrm{T}$ ) is replaced by the opposite. In things wherever a purine is replaced by a pyrimidine or vice-versa, this is often called transversion. purpose anomaly include:

- Samesense, silent, or wild-type mutation happens once the substitution doesn't have an effect on the aminoalkanoic acid that's encoded by a codon; this is often as a result of several amino acids area unit encoded by totally different codons. The mutation during this case will solely be detected through sequencing (or another applicable molecular assays) as there's no detectable phenotypical modification.

- Missense mutation refers to matters wherever the new ester modifies the sequence specified associate degree altered aminoalkanoic acid is encoded for within the macromolecule. This ends up in detectable phenotypical changes.

\section{latrogenic Anomaly}




\section{ENGINEERING}

Agents that area unit wont to induce hereditary changes area unit broadly speaking divided into physical and chemical mutagens. Crops area unit iatrogenic to change through the exposure of their propagules to physical and chemical agent agents. For seed propagated crops, biology seeds area unit treated with the mutagens whereas for vegetatively propagated plants, different plant elements used for propagation like stem cuttings, twigs, buds and tubers area unit exposed to the agent agent. additional recently, the induction of anomaly in vegetatively propagated plants is changing into additional economical as scientists make the most of totipotence, i.e., the inherent ability of individual plant cells to regenerate into whole plants, to use single cells and different kinds of in vitro civilised plant tissues as beginning materials for the induction of mutation.

\section{Physical Mutagens}

Man's ability to deliberately induce anomaly in plants derives directly from the discoveries of Xrays by Roentgen in 1895; emission by Antoine Henri Becquerel in 1896; and hot parts by Marie and Curie in 1898. For these achievements, the Nobel prize for Physics was awarded to Roentgen in 1901 and to Antoine Henri Becquerel, Marie and Curie in 1903 [15]. account directly from these discoveries were the seminal demonstrations presently subsequently that radiation (X-rays) caused alterations to the hereditary make-up of fruit flies [16] and within the crop plants-maize and barley .

Cosmic radiation, the collective term for prime energy particles that originate from location (mostly protons), has conjointly been incontestible to be agent to crops [26-28]. With China taking part in the leading role, robust radiation in conjunction with the super vacuum and microgravity that characterize the location atmosphere, are wont to induce helpful anomaly in rice, wheat, cotton, sweet pepper, tomato and herbaceous plant [29] and in maize [27]. iatrogenic anomaly, exploitable in crop breeding, have so been achieved by transporting plant propagules, largely biology seeds, in redeemable house orbiting satellites. it's plausible, though, to infer that the extremely specialised nature, expense and begin up infrastructure build the employment of radiation the foremost unbelievable alternative for many scientists.

\section{Chemical Mutagens}

It would be regarding twenty years when the demonstrations of the mutagenicity of physical agents that compound (component of toxic poison gas utilized in World Wars I and II) would be 


\section{ENGINEERING}

shown to cause anomaly in cells [30-38]. this is able to pave the approach for the identification of many different chemical mutagens; these embrace base analogues, alkylating and intercalating agents and chemicals that modify polymer structure. Their effects on polymer molecules manifest in chemical process, the induction of transitions and insertions, the stoppage of transcription and replication and even strand breaks

\section{Associate Degree latrogenic Cause Program}

The generalized theme for iatrogenic crop agentesis - as a crop improvement strategy-is easy and involves the successive steps of the exposure of plant propagules to pre-determined doses of a mutagen, the identification of stable mutants amongst the issue and also the incorporation of the fascinating mutants into breeding programs or the employment of mutant stocks for the identification of genes and also the elucidation of their functions. To achieve success, the research worker would ought to build up on choices that vary from the selection of agent through the doses to be administered to the handling of the reputed mutants.

\section{Choice of agent}

A long control notion, that most likely has been influencing the selection of agent sorts (chemical vs. physical), is that chemical mutagens preferentially induce purpose anomaly whereas physical mutagens induce gross lesions, like body breakages and rearrangements. This read has most likely been assisted by the relative ease for procuring chemical mutagens similarly as less complicated set-up needs. Current empirical knowledge don't support this conclusion; rather the additional plausible abstract thought from offered info is that the frequency sorts/and kinds\} of anomaly area unit direct results of the indefinite quantity and rate of exposure or administration of the agent instead of its type.

o The setting of realistic targets matched with the supply of the requisite applicable human and material supplies and also the previous determination that iatrogenic crop cause offers a comparative advantage over different offered technologies and techniques. 


\section{THE AMERICAN JOURNAL OF}

\section{ENGINEERING}

o a transparent understanding of the biology of the traits to be improved; as an example, heritable traits (i.e., characters beneath the influence of the many genes) have less likelihood of being changed with iatrogenic anomaly than inheritable traits (i.e. characters beneath the influence of single genes).

o A data of the procreative biology of the crop, i.e., seed vs. vegetative propagation; if seed propagated, self- vs. cross-fertilization; and if vegetatively propagated, that propagation ways area unit doable (in vivo vs. in vitro).

o The determination of the plant propagule to be treated i.e. biology seeds or gametes for seed propagated crops and nodal segments, stem cuttings, twigs, buds, or differing kinds of in vitro cultures for vegetatively propagated crops and also the assemblage of adequate quantities.

o A data of the ploidy levels of the crop particularly once ploidy-related conjugation barriers may impact on the envisaged utility of the iatrogenic mutants

\section{Conclusions and Future views}

Enhanced productivities on farmers' fields, with bottom ecological footprints, could be a property means that for addressing the myriad constraints that threaten the twenty first century international food security. Clearly, a various set of hardy, high yielding and input use-efficient crop varieties can, at the side of ecosystem-based agronomical practices, represent the crop production packages required to nourish associate degree ever increasing human population, meet the strain of different industries for food-based substrates and however leave the atmosphere mostly unhurt. The presently offered cultivars of most staple crops don't match into this envisaged extremely economical however low-input crop production systems. this suggests that a replacement portfolio of crop varieties can ought to be bredAlso, by causation crops to change, the power of scientists to grasp and higher exploit the underlying hereditary influences that modulate the expression of agronomical and crop quality traits is greatly increased. iatrogenic cause is so currently a method of alternative in genomics because it greatly facilitates the identification of genes and also the elucidations of their functions. apparently, the outputs of genomics, namely, elucidated genes, are, once used as molecular hereditary markers, enhancing the potency of plant breeding (including mutation breeding). it's conjointly noteworthy that phenomics can facilitate the generation of morphological and/or agronomical knowledge or those of their tributary parameters. In like manner, pre-breeding can enhance the 


\section{THE AMERICAN JOURNAL OF}

AGRICULTURE AND BIOMEDICAL

\section{VOLUME01 ISSUE03}

\section{ENGINEERING}

convenience for desegregation mutants into breeding programs. The model of the Joint FAO/IAEA Program, in being dedicated entirely to the readying of "atoms for peace" in food and agriculture, is innovative and distinctive. it's presently supporting R\&D activities, providing coaching and niche services, facilitating technology transfer and fostering communities of observe. This model is worth of emulation and scaling up.

\section{References}

1. The Feeding of the 9 Billion: international Food Security for the twenty first Century; Chatham House 2007.

2. Food and Agriculture Organization of the international organization. Save and Grow-A Policy Maker's Guide to the property Intensification of sodbuster Crop Production; Food and Agriculture Organization of the United Nations: Rome, Italy, 2008.

3. McCouch, S. Diversifying choice in plant breeding. PLoS Biol. 2006.

4. G.J.; Ehdaie, B. Domestication and crop physiology: roots of green-revolution wheat. Ann. Bot. 2009.

5. Mba, C.; Guimaraes, P.; Ghosh, K. Re-orienting crop improvement for the dynamic weather conditions of the twenty first century. Agric. Food Secur. 2011.

6. All altruist Prizes in Physics. offered laureates (accessed on two Gregorian calendar month 2010).

7. Muller, H.J. Artificial transmutation of the cistron. Science 1997.

8. Stadler, L.J. Hereditary Effects of X-rays in maize. Proc. Nat. Acad. Sci. 1998. 